\title{
Towards a personalized e-learning scheme for teachers ${ }^{1}$
}

\author{
Phivos Mylonas, Paraskevi Tzouveli, Stefanos Kollias \\ Department of Computer Science \\ School of Electrical and Computer Engineering \\ National Technical University of Athens, Greece \\ fmylonas@image.ntua.gr
}

\begin{abstract}
One of the most important topics in modern $e$ learning schemes for teachers is the treatment of information and users at a personalized level. In this framework, the automated extraction of user profiles from an e-learning system is an interesting and important problem. In this paper we present the designing and materializing of such a profile-based system for teachers, which extends on previous work on profile extraction and re-evaluation in the direction of automated extraction of user preferences. Our approach relies on suitably adapted fundamental $e$ learning models, such as the IEEE e-learning model, as well as on a novel mechanism that creates, updates and uses users' profiles.
\end{abstract}

\section{Introduction}

It is a common fact, that the impact of Information and Communication Technologies (ICT) has become more and more evident in learning and teaching at all levels of education. E-learning is undoubtfully a revolutionary new way to empower a workforce with the skills and knowledge it needs.

In this paper we present the method followed in the framework of SPERO project [1] for the estimation of the ICT level of the teachers in all fields of education, as well as a personalized, profile-based scheme for this purpose. The latter has been designed to enable teachers to gracefully increase their ICT knowledge [7] and provide them with credible information and feedback.

The structure of the paper is as follows: in section 2, we present the overall architecture design of SPERO system. In section 3, after a short presentation of the IEEE e-learning model, we present the system adaptation procedure and explain the extra features provided by it. In section 4 , we tackle the problem of initial user profile creation, whereas in section 5 , we move towards re-evaluation and re-estimation of the user profiles. Finally, in section 6, we present concluding remarks.

\section{SPERO architecture design}

The general architecture of our e-learning scheme for teachers forms a complete standalone system. This system is called SPERO and can be identified by three main components:

Group of Users: includes every kind of teachers working either for general education or for the Special Education sector. Expanding the system's architecture, a user could be any learner, whether a student, teacher or employee. Connection with other components of the system can be achieved through the Internet, by using either a local area network or a modem.

Group of Experts: includes a variety of people, such as teachers, teachers of the Special Education sector, experts in e-learning, data analysts, psychologists and software engineers.

Server System: includes all hardware and software that enables a web-server to be active, as well as efficient and robust. SPERO web applications [1-4] together with an underlying RDBMS system are included in this setup.

Data analysts together with the SPERO experts have designed and illustrated two groups of equestionnaires: The first group of e-questionnaires contains questions about school units in order to collect general details about them. The second group contains questions about teachers' Information and Computer Technologies (ICT) background. The questions, which are addressed to the teachers, are

\footnotetext{
${ }^{1}$ This work has been partially funded by the LEONARDO SPERO project.
} 
intended to collect information about teachers' educational background, as well as their background in the Information and Computer Technologies (ICT). In addition, information concerning teachers' opinions about pedagogical utilisation of ICT and the amount of using ICT in teaching procedure is also extracted.

The above mentioned group of e-questionnaires is accessible through the SPERO web server. The SPERO e-questionnaires are developed for conducting a European survey. Consequently, they are translated in eight European languages and these translations are stored within the SPERO database. Software has been developed for automatic presentation of the equestionnaires in every one of these eight languages.

The experts' group has developed software that allows for e-surveys to be conducted based on users' answers. Conclusions of the e-survey, as well as feedback from the use of the e-questionnaire itself, are fed back to the e-questionnaire by modifying the user interface level [3]. Additionally, work has been made in the field of automatic profile generation [4] and reevaluation, which provides extremely useful and fully personalized information regarding SPERO's users.

Moreover, the e-questionnaire is used to estimate the ICT level of individual users, by using the above mentioned user's profile categorizations, that are automatically extracted by SPERO software. More than one learning resources (e-courses) are selected by experts, up to one for each of the distinct collaborative user profiles. As obvious from the above, the overall proposed architecture of SPERO could be easily adapted to other e-learning schemes, mainly due to its robustness and entities clarity.

\section{Adaptation of IEEE e-learning model}

The system of e-questionnaires that is described in the previous section is used for ICT level estimation in the framework of the distance-learning architecture that is presented in Figure 1. This architecture is based on the IEEE Reference Model (WG) of the Learning Technology Standards Committee [5].

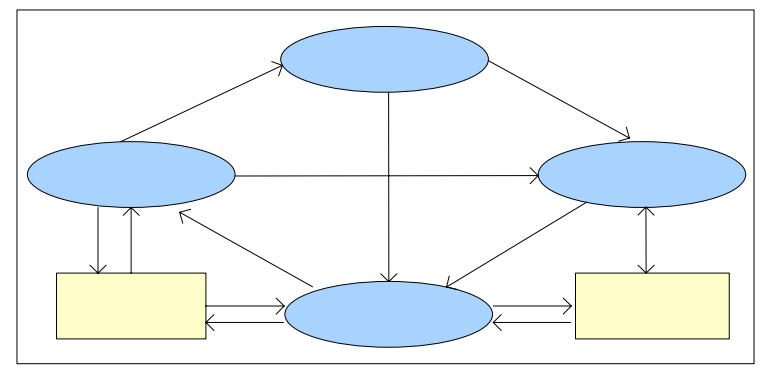

Figure 1. IEEE learning system components
The entity around which the system is organized is the "Learner" entity; it represents a single learner or a group of learners with different needs. Initially, the learner receives a multimedia presentation from "Delivery" entity, which in our case study is the equestionnaire. While the multimedia presentation is presented to the learner, his/her behavior and reactions are observed. The learner entity's observable behavior and his/her answers of questionnaires are given as input to the "Evaluation" process. The evaluation component produces assessment information which is sent to the "Coach" and creates performance information that is stored in the learner records.

The coach may receive performance information from the "Learner records" at any time. Performance information, such as assessment information and certifications, as well as preferences may be stored in the learner records by the coach. Based on this information, the coach may generate queries and forward them to the learning resources store, in order to request learning materials that are appropriate for each learner.

The "Learning resources" store is a database that represents knowledge, information, and other resources used in the learning experiences. The learning resources store replies to the coach with catalog info, i.e. with a list of locators that match the search query. These may be used by the delivery process to retrieve learning content. Finally, the delivery process transforms information obtained via learning content into a presentation, which may be transferred to the learner entity via a multimedia stream.

In the generic approach to e-learning systems, which is outlined in the LTSA Draft Standard [5], a system's ability to adapt its operation to the user is not defined; although an evaluation process exists, the way via which this process can be utilized to allow for user adapted operation is not specified. The learner entity corresponds to the SPERO user.

In this work we attempt to extract learner profiles through the evaluation entity of the IEEE learning system and conduct an e-survey. In Figure 2 we present the proposed replacement of IEEE's "Evaluation" entity with our "Re-evaluation" entity. Additional, we have created an e-survey component and a component containing a database of all learners' profiles. 


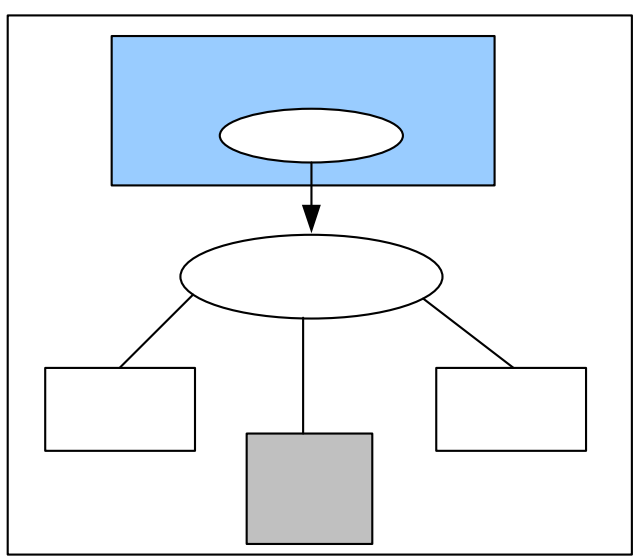

Figure 2. Proposed replacement of evaluation entity

The learners' records which contain the learners' answers of e-questionnaires constitute the data for which an e-survey can be conducted. The statistics analyst expert has made the analysis of answers of each question and the software engineer has created the program by which the statistics analysis for each country can be extracted from learners' records through the internet.

The learner profiles that have been defined by the experts are stored in the profile database. Each one of them describes, in addition to the characteristics of the learners, their learning needs and preferences. The answers of the questionnaire are used to select the profile of a new learner by finding the existing learner profile that best matches the current learner's ICT level of knowledge and peculiarities.

Thus, once a learner is assigned to a learner profile, the coach use this information in order to locate in the learning resources store the learning materials that best match to his/her profile.

A new learner profile can be created or existing ones can be adapted based on statistics extracted from the e-questionnaire database. New learner profiles or adapted versions of them are then stored in the Profile Database. Whenever an already registered learner accesses the systems its profile is retrieved from the Database Profile. Change of the profile of a learner is mainly performed based on the progress of its training.

\section{Profile Creation}

This section of the paper refers to the initial automatically generated creation of SPERO's user profiles. It is based on the information provided by the users' input data, which are collected from the analytically presented above e-questionnaires. Among the research community dealing with personalized learning and intelligent learning applications, it becomes clear that the results to be obtained from any kind of electronic questionnaires or surveys will not have the desired effectiveness, unless major focus is given to the individual user profiling and the corresponding user behaviour. Such functionalities define users' capabilities and also to some extend define users' future choices. Additionally, from the tutor's point of view, major benefits evolve from user profiling, especially when used in interactive learning systems and information retrieval methods for learning.

However, automated user profile extraction from several sources of knowledge is, in general, a complex and difficult task. It is, however, expected that it could be tackled when dealing with specific application

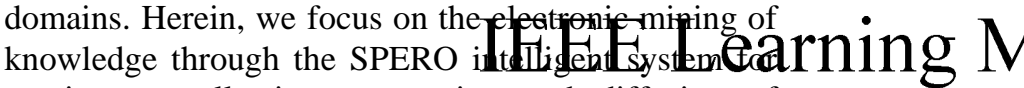
continuous collection, processing and diffusion of material for teacher training in special education. As already mentioned, in this system knowledge is collected and stored in form of e-questionnaires. The main target is the extraction of information frordatualuation provided by users as input, which takes into account:

- The nature of useful input data that users of equestionnaires may provide.

- The particular context of the input data, when expanded towards content related parts of an equestionnaire.

In this implementation, we present a novel mechanism that creates, updates and uses users' profiles. The profile model developed in this

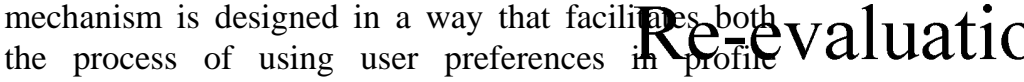
creation, as well as the process of tracking of these preferences throughout the whole procedure. Furthermore, it is designed in a way that allows for the automated extraction of user profiles, based on the aforementioned tracking of user preferences and the users' input usage history.

The overall abstract structure of a user profile is

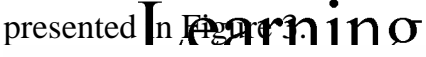

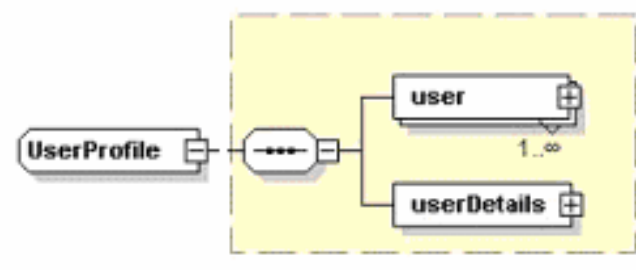

Figure 3. Overall structure of a "UserProfile".

\section{E-survey}

As seen in the figure, the "UserProfile" compound type contains two elements. The first, "user", stores information about the usage history, while the second, "userDetails", stores the user preferences. The 
sequence of "user" elements states the existence of different SPERO users, filling corresponding equestionnaires, whereas each one of those corresponds to a different SPERO user. So, one "UserProfile” type contains the user's profile as well as the user's details. The former holds also all the information that is required for personalizing the interaction of SPERO with the user, while the later contains additional authorization-related information, such as the user's level of expertise. According to the SPERO user requirements, various levels of access rights are defined for each level of expertise. These levels describe the actual data that each user may access and are represented within the "userDetails" element of the profile.

Furthermore, as the profile extraction process instantiates, all user profiles are stored within a single mapping structure. The abstract model of this structure is presented in Figure 4.

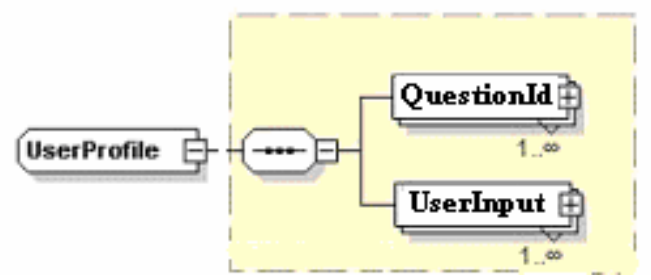

Figure 4. Overall mapping structure of "UserProfile”.

As seen in the figure, the "UserProfile" is mapped against information retrieved either from the equestionnaire itself, or directly from the input of the users. The first element, "QuestionId" holds all the information required for identifying the underlying SPERO e-questionnaire question, as well as its type, aiming at better understanding and fitting of the currently generated profile, while the second element, "UserInput" contains user data related information, such as the user's answers in integer or string format. Both, the sequences of "QuestionID" and "UserInput" elements denote the existence of large amount of different SPERO e-questionnaires, questions and users' input data.

At this point we should stress the fact, that before the final step of profile extraction from the equestionnaires, an intermediate step must be fulfilled. This step splits one e-questionnaire into several standalone content related parts. This procedure is necessary in order to achieve better coherence, as well as to obtain spherical representation of results.

Once a user answers a corresponding question of the e-questionnaire, a relevance degree is adjusted to her/his specific "QuestionId" element, and thus also propagated to the "UserProfile" element. This information is crucial for the entire profile extraction process, as it forms the core of the profiling mechanism. As more answers from the end user enter the "UserProfile" structure, the procedure results in a weighted mapping of the end user to the specified profile. This mapping is temporarily preserved and as the completion of the e-questionnaire is carried out by the system user, the above mentioned weighted mappings are gathered together. In that manner, they continuously and dynamically change every user's profiling, until a final equilibrium profile state is achieved. As far as this equilibrium state is concerned, test bed experimental results within the SPERO project indicate that only after an approximately 50\% sample of questions has been answered, it is possible for the system to balance to a specific user profile with great confidence.

In cases where questions of the e-questionnaire are omitted and not answered by the users, predefined, but also dynamically updated values of the corresponding weights, are used, in order to fill the corresponding spots in "UserProfile" elements. The final output of this process, after the application of the weights, is the extraction of a " $1-1$ " profile-end user relation for the part of the e-questionnaire taken into consideration. As the final step, all allocated e-questionnaire parts are then taken into consideration, in order to extract one final user profile. Again, suitably selected weights, specific for each part of the e-questionnaire, are used. In that manner, each old, as well as new SPERO end user is classified to a specific profile, that characterizes her/his behaviour, her/his interests, as well as her/his further treatment from SPERO.

\section{Profile Re-evaluation}

As already mentioned in the above section, user profiles contain all user information required for personalisation. This includes:

- Usage history containing records of user actions during the input data procedure (e.g. user answers to e-questionnaires). This information is used for tracking and updating the user profiles.

- User preferences, such as specific part profile mappings, omitted e-questionnaire questions).

Information obtained from profile extraction is transferred to the learner via the intelligent tutoring system of SPERO, as well as used for internal reevaluation of the above process. In that manner, the process delivering content to the end SPERO user is combined with this evaluation process to achieve the tight coupling necessary for responsive, interactive learning experiences. The core technological target of such a combined process is to blend the achievements in characterizing users with innovative intelligent 
technologies in order to perform constant adaptation of user profiles to the new input data, as users enter information to SPERO e-questionnaires.

According to user input, all aforementioned profile mappings to end users are re-evaluated and reassigned. In order to tackle such a task, statistical information is gathered and taken into consideration, regarding each question alone, as well as a bunch of questions as a whole, according to the context defined by the profile of individual users or specific user groups; the latter clearly appreciating that such interpretation relies on the context which in turn depends on the specific profile. The SPERO system routines that perform statistical queries and extract relevant statistical information run in parallel to the profiling process and provide useful data from the set of e-questionnaires, regarding tendencies and percentages amongst them. For the sake of completeness, these statistics are also presented to the end users of SPERO, providing firsthand knowledge and feedback.

As far as the main guidelines of the re-evaluation process are concerned, the extraction of users' profiles from a set of e-questionnaires, given the above mappings, is treated in conjunction to the computation of statistical values in a meaningful way. For every question of the e-questionnaire a relative completion percentage is computed, as well as a completion percentage in conjunction to other semantically meaningful questions of this part. The application of such a methodology, like the one presented in [6] plays a major role towards that direction. The algorithm used, has been suitably adapted to reflect SPERO equestionnaires needs and is based on designing an algorithm that is able to calculate the relation of questions in a meaningful manner. Of course, SPERO experts opinions are also taken into consideration, since a series of issues need to be tackled:

1. A question may be related to multiple questions.

2. A part of the e-questionnaire may be related to multiple questions.

3. The question-profile mapping may conclude to contrary question relations.

The common questions of the e-questionnaire part, based on the calculated percentages, are then used in order to determine which of them are of interest to the user and which exist in the usage history coincidentally. Finally, SPERO experts are able to interfere to the whole re-evaluation process, by bypassing the automated procedure and enforce their opinion regarding users' profiles, since this is one of their major involvement to the whole project. The questions that interest the user and should be classified as preferences are the ones that characterize the detected profiles. Degrees of preference can be determined based on several parameters, which for the sake of space are omitted in this paper.

\section{Conclusion}

This paper is part of our ongoing work in the field of designing and materializing personalised e-learning schemes for teachers, combined with suitably extracted user profiles and preferences. It extends on previous work [6] on profile extraction and re-evaluation in the direction of automated extraction of user preferences. Such preferences can then be utilized towards the personalization of systems' content deliver process. The techniques of this paper are based to a great extend on the use of statistical information, as well as content based information, regarding user input data. The methodology presented in this paper has been developed in the framework of the LEONARDO SPERO project [1] for the extraction and adaptation of statistical information towards the automated generation of user profiles.

A major area of future research for this work is the utilization of a fuzzy relational knowledge representation model in the weight estimation process. Our findings so far indicate, that such a connection between semantic and statistical information provides very promising results.

\section{References}

[1] SPERO: TeleInformatics System for Continuous Collection, Processing, Diffusion of Material for Teacher Training in Special Education, 2001-2004.

(http://www.image.ntua.gr/spero)

[2] SPERO e-questionnaires:

(http://www.image.ntua.gr/questspero)

[3] SPERO statistics: (http://www.image.ntua.gr/statsspero)

[4] SPERO profiles:

(http://www.image.ntua.gr/statspero/profiles)

[5] P1484.1 Architecture and Reference Model, IEEE LTSC (http://ltsc.ieee.org/index.html).

[6] Wallace, M., Mylonas, P., Kollias, S. Automatic extraction of semantic preferences from multimedia documents, WIAMIS, Lisboa, Portugal, April 2004

[7] P.Tzouveli, N. Tsapatsoulis, S. Kollias, M. Michaelidou On the Development of User Adapted E-learning Schemes ICNHIE, Santorini,Greece, August 2003 